\title{
A Família no contexto da hospitalização: revisão sistemática
}

\section{The family in the context of hospitalization: a systematic revision}

\section{La familia en el context de hospitalización: revisión sistemática}

\author{
Adriano Valério dos Santos Azevêdo* \\ Universidade Federal de Santa Catarina - UFSC, Florianópolis, Santa Catarina, \\ Brasil
}

\author{
Maria Aparecida Crepaldi** \\ Universidade Federal de Santa Catarina - UFSC, Florianópolis, Santa Catarina, \\ Brasil
}

\section{Carmen Leontina Ojeda Ocampo More***}

Universidade Federal de Santa Catarina - UFSC, Florianópolis, Santa Catarina, Brasil

\begin{abstract}
RESUMO
A pesquisa objetivou apresentar uma revisão sistemática de artigos científicos sobre a família no contexto da hospitalização. Nas bases de dados (Lilacs, Medline, IBECS, PsyclNFO, Wiley Interscience, Pubmed, Web of Science) buscou-se selecionar os estudos empíricos publicados entre 20072012. Foram construídas categorias para analisar estes estudos: família e criança hospitalizada, família e adulto hospitalizado, família e idoso hospitalizado. Estas pesquisas enfatizam a vivência de familiares, avaliação de estresse e estratégias de enfrentamento, e a relação equipe de saúde e família. Foram identificados maior número de publicações sobre família e criança hospitalizada, e estes estudos apresentam as alterações que ocorrem na dinâmica familiar, diante da situação de doença e hospitalização. Existe a necessidade da atenção integral à família do paciente hospitalizado para promover o acolhimento e minimizar o sofrimento ocasionado pelo processo de hospitalização. Estes resultados das pesquisas sobre famílias de pacientes hospitalizados auxiliam a construção de propostas de avaliação e intervenção psicológica.

Palavras-chave: família, internação, criança, cuidadores, ciclos de vida familiar.
\end{abstract}

\section{ABSTRACT}

The research aimed to present a bibliometric study of scientific articles about the family in the context of hospitalization. From queries in databases (Lilacs, Medline, IBECS, PsycINFO, Wiley Interscience, Pubmed, Web of 
Science) was able to select the empirical studies published between 20072012. Categories were built to analyze these studies: family and child hospitalized, family and adult hospitalized, family and elderly hospitalized. These studies emphasize the family experience, evaluation of the levels of stress and coping strategies, and relationship team of health and family. The studies highlight changes that occur in family dynamics on the disease situation and hospitalization of a member. There is a need for comprehensive care family of hospitalized patients to promote the acceptance and minimize the suffering caused by the process of hospitalization. These results of research on families of hospitalized patients build proposals for evaluation and psychological intervention.

Keywords: family, internment, children, caregivers, family life cycle.

\section{RESUMEN}

La investigación pretende presentar un estudio bibliométrico de los artículos científicos sobre la família en el contexto de hospitalización. De las consultas sobre bases de datos (Lilacs, Medline, IBECS, PsyclNFO, Wiley Interscience, Pubmed, Web of Science) fue posible seleccionar los estudios empíricos publicados entre 2007-2012. Categorías fueron construidos para analizar estos estudios: familia y niño hospitalizado, familia y adulto hospitalizado, familia y personas de edad avanzada hospitalizados. Estos estudios enfatizan la experiencia de los familiares, la evaluación de estrategias de estrés y afrontamiento y relación equipo de salud y familia. Mayor cantidad de publicaciones_sobre la familia y el niño hospitalizado, y estos estudios ponen de relieve los cambios que ocurren en la dinámica familiar ante la situación de enfermedad y hospitalización. Estos resultados de la investigación sobre las familias de los pacientes hospitalizados ayudan a construir propuestas para la evaluación e intervención psicológica.

Palabras clave: familia, hospitalización, niños, cuidadores, ciclo de vida familiar.

\section{I ntrodução}

A família representa um sistema aberto que cumpre funções e enfrenta determinadas tarefas desenvolvimentais, que exigem adaptação e reestruturação (Minuchin, 1990). Nessa perspectiva sistêmica, a socialização, por exemplo, representa uma dessas tarefas, ao considerar que no processo de desenvolvimento humano, todos os integrantes do ambiente familiar são influenciados pelas mudanças que ocorrem no decorrer das interações (Dessen \& Braz, 2005).

A noção de sistema parte do princípio de que os integrantes estão em constante interação compartilhando crenças e desenvolvendo formas de comportamento por meio de interrelações. Assim, as ações e comportamentos de um de seus membros influenciam 0 funcionamento do sistema e a dinâmica familiar.

O sistema familiar, para a perspectiva estrutural da terapia familiar, apresenta subsistemas que delimitam diferentes funções, por exemplo, o subsistema conjugal integra a união de dois adultos com o propósito de formar uma família, o subsistema parental possibilita 
que os pais desenvolvam os cuidados básicos com os filhos, assim como o subsistema fraternal que inclui a dinâmica estabelecida pelos irmãos (Minuchin, 1990). Os subsistemas são formados por uma pessoa ou díade, o que permitem o desenvolvimento de habilidades, a organização de tarefas, e a diferenciação das funções que são estabelecidas. As fronteiras representam o conjunto de normas que organizam a dinâmica do grupo familiar, definem as funções, e orientam o estabelecimento de princípios de moralidade (Minuchin, 1990).

A definição do conceito de família relaciona aspectos multifatoriais que estão em constante interação, assim para entender 0 funcionamento da família é necessário investigar as relações desenvolvidas pelos seus integrantes. Nesse contexto, as pesquisas cumprem um papel fundamental quando investigam a dinâmica familiar e os aspectos específicos de cada ambiente. No que se refere aos estudos com famílias, pesquisadores apontam a importância de delimitar o objeto de estudo, a perspectiva teórica, as estratégias de investigação, a seleção dos instrumentos e a análise de dados (Böing, Crepaldi, \& Moré, 2008). Estas orientações facilitam iniciar pesquisas com um enfoque científico para investigar temáticas complexas, e com isto apresentar novos estudos sobre famílias.

Diante das possibilidades de investigação, destacam-se os estudos sobre famílias que se encontram vivenciando situações de adoecimento e hospitalização. Tais pesquisas enfatizam a recepção da família na hospitalização de crianças com doenças crônicas (Crepaldi \& Varella, 2000); a experiência da família de um adulto hospitalizado com doença crítica (Eggenberger \& Nelms, 2007), avaliação dos indicadores de estresse e enfrentamento de acompanhantes de pacientes adultos hospitalizados (Chui \& Chan, 2007). A comunicação entre profissionais de saúde e família (Aein, Alhani, Mohammadi, \& Kazemnejad, 2011; Nieweglowski \& Moré, 2008). Estas pesquisas indicam que a hospitalização de um membro do grupo familiar provoca uma série de transformações relativas às mudanças na rotina de vida, que exigem o manejo adequado para facilitar o enfrentamento.

A revisão de estudos sobre família e hospitalização de seus integrantes possibilita iniciar o mapeamento com o propósito de identificar as temáticas específicas, os participantes destes estudos, e os resultados que foram obtidos, para fins de verificar as possibilidades de pesquisas, e de aprimoramento de aspectos relevantes do tema de estudo. Justifica-se a relevância da investigação de pesquisas realizadas com famílias para fomentar a reflexão da dinâmica familiar que se estabelece em torno do adoecimento, o que permite auxiliar os profissionais de saúde nas relações interpessoais com estas famílias. $\mathrm{Em}$ relação às contribuições científicas, isto promoverá a sistematização dos 
resultados desses estudos visando elaborar programas e protocolos de intervenção psicológica, o que permite refletir sobre estratégias de pesquisa a serem utilizadas no contexto hospitalar. Assim, esta pesquisa tem por objetivo apresentar uma revisão sistemática de artigos científicos sobre a família no contexto da hospitalização.

\section{Método}

Trata-se de uma revisão sistemática, que se refere a um estudo de fontes primárias, este que objetiva realizar o mapeamento e a avaliação crítica de resultados de pesquisas, relacionado a um tópico que se pretende investigar, e o agrupamento desses estudos para uma compreensão geral (Dresch, Lacerda, Antunes-Júnior, 2015). É por meio do processo de revisão sistemática, orientado por etapas metodológicas que possam ser utilizadas por outros pesquisadores, que se identifica o panorama dos estudos que foram realizados, e com isso torna-se possível a análise e a reflexão crítica, visando promover avanços na construção do conhecimento.

Foram realizadas consultas nas bases de dados, Bireme, Lilacs, Medline, IBECS, PsyclNFO, Pubmed, Wiley Interscience, Web of Science - utilizando as palavras-chave família no hospital, family in the hospital, família hospitalizada, family hospitalized. As consultas nessas bases de dados foram realizadas no mês de dezembro de 2012. Procurou-se incluir os artigos publicados no período de 20072012, que se referiam aos relatos de pesquisas empíricas sobre famílias de pacientes no hospital geral. Os estudos teóricos, as dissertações e teses foram excluídos. Foram selecionados os artigos em português e inglês para apreciação dos resumos e trabalhos completos. Os artigos foram analisados e delimitados quanto aos temas gerais e específicos, ano de publicação, áreas de conhecimento, periódicos científicos, e os métodos utilizados nas pesquisas, assim foi possível apresentar a descrição quantitativa e qualitativa. Utilizou-se a análise temática para a identificação dos conteúdos dos artigos, por meio da leitura do título e objetivo, o que representou a primeira fase de categorização, em seguida os artigos foram classificados em categorias e subcategorias específicas, de acordo com o conteúdo que foi investigado (Bardin,1979).

\section{Resultados}

Identificaram-se 135 artigos científicos. A frequência da produção internacional é superior, se comparada aos estudos nacionais. Foram construídas três categorias para representar os artigos pesquisados: 1. Família e criança hospitalizada (artigos sobre a hospitalização de 
crianças e a experiência de seus familiares), 2. Família e adulto hospitalizado (artigos sobre a hospitalização de adultos e os cuidadores familiares), 3. Família e idoso hospitalizado (artigos sobre a hospitalização do idoso e seus familiares). A Tabela 1 apresenta dados sobre o ano de publicação e frequência de artigos publicados sobre as categorias que foram construídas. A categoria família e criança hospitalizada apresenta maior frequência de artigos publicados, o que permite ressaltar que se trata de uma área de interesse dos pesquisadores.

Tabela 1. Ano de publicação e frequência de artigos publicados $(\mathrm{N}=135)$

\begin{tabular}{cccc}
\hline Ano & $\begin{array}{c}\text { Família e criança } \\
\text { hospitalizada }\end{array}$ & $\begin{array}{c}\text { Familia e adulto } \\
\text { hospitalizado }\end{array}$ & $\begin{array}{c}\text { Família e idoso } \\
\text { hospitalizado }\end{array}$ \\
\hline 2007 & 16 & 8 & 1 \\
2008 & 22 & 6 & 0 \\
2009 & 23 & 4 & 2 \\
2010 & 15 & 3 & 0 \\
2011 & 20 & 5 & 1 \\
2012 & 4 & 3 & 2 \\
Total & 100 & 29 & 6 \\
\hline
\end{tabular}

A Tabela 2 apresenta a frequência de publicações nacionais e internacionais, e de uma maneira geral, é possível verificar maior concentração de estudos que se referem à família e criança hospitalizada.

Tabela 2. Frequência de publicações nacionais e internacionais $(\mathrm{N}=135)$

\begin{tabular}{lcc}
\hline Categorias & \multicolumn{2}{c}{ Publicações $(\mathrm{N})$} \\
\hline Familia e criança hospitalizada & Nacionais & Internacionais \\
\cline { 2 - 3 } Familia e adulto hospitalizado & 11 & 55 \\
Familia e idoso hospitalizado & 4 & 18 \\
Total & 60 & 75 \\
\hline
\end{tabular}

A Tabela 3 apresenta as áreas de conhecimento responsáveis pelas publicações de artigos sobre a família no contexto da hospitalização. Esses estudos estão concentrados na área da enfermagem e nas demais áreas das ciências da saúde. 
Tabela 3. Frequência de publicações por área de conhecimento $(\mathrm{N}=135)$

\begin{tabular}{lr}
\hline Áreas & Frequência (n) \\
\hline Enfermagem & 104 \\
Ciências da Saúde (médicas/biológicas) & 12 \\
Cuidado e saúde da criança (interdisciplinar) & 8 \\
Psicologia & 6 \\
Pediatria & 5 \\
Total & 135 \\
\hline
\end{tabular}

A Tabela 4 destaca as categorias das pesquisas realizadas com famílias de pacientes hospitalizados, e a frequência referente ao método de pesquisa utilizado nestes estudos. Verificou-se na categoria família e criança hospitalizada maior quantidade de pesquisas que utilizaram o método qualitativo por meio de entrevistas. Nos estudos sobre estresse e enfrentamento, identificouse a utilização do método quantitativo por meio de escalas. Na categoria família e adulto hospitalizado, os estudos sobre a experiência da família utilizaram entrevistas, o que também foi possível verificar nas pesquisas sobre família e idoso hospitalizado.

Tabela 4. Frequência referente aos métodos e instrumentos utilizados nas pesquisas $(\mathrm{N}=135)$.

\begin{tabular}{lrr}
\hline Categorias & $\begin{array}{c}\text { Método Quantitativo } \\
\text { (Questionários/Escalas) } \\
\text { Frequência (N) }\end{array}$ & $\begin{array}{c}\text { Método Qualitativo } \\
\text { (Entrevistas) } \\
\text { Frequência (N) }\end{array}$ \\
\cline { 2 - 3 } Familia e criança hospitalizada & 17 & 39 \\
Vivências de familiares & 13 & 1 \\
Estresse e enfrentamento & 3 & 27 \\
Familia e equipe de saúde & 33 & 67 \\
Total & & \\
Familia e adulto hospitalizado & 8 & 12 \\
A experiência da família & 4 & 1 \\
Estresse e enfrentamento & 2 & 15 \\
Família e equipe de saúde & 14 & 2 \\
Total & & 2 \\
Familia e idoso hospitalizado & 1 & 4 \\
A relevância da familia & 1 & 2 \\
Familia e equipe de saúde & 2 & \\
Total & & \\
\hline
\end{tabular}




\subsection{Família e criança hospitalizada}

Os estudos sobre a família da criança hospitalizada evidenciam as vivências dos familiares, o estresse, enfrentamento e suporte familiar, e a relação família e equipe de saúde. Estas pesquisas serão contextualizadas por meio das principais informações com o propósito de apresentar sínteses dos resultados obtidos.

\subsubsection{Vivências de familiares}

As pesquisas investigaram as experiências de mães acompanhantes de crianças hospitalizadas na unidade neonatal e no setor de oncologia. Outros estudos estudaram as necessidades de mães que acompanham os filhos hospitalizados, a vivência na unidade de terapia intensiva pediátrica, no pronto-socorro, e no grupo psicoeducativo no contexto hospitalar. O estudo de Arruda e Marcon (2007) objetivou compreender como foi para a família em expansão conviver com as intercorrências durante a gestação e o parto do bebê nascido prematuro e com muito baixo peso. Verificou-se que as intercorrências vivenciadas no período gestacional apresentam repercussões na dinâmica familiar, e quando as complicações provocam o parto prematuro, surgem sentimentos e angústias que ocasionam sofrimento e dificuldades relacionadas à assistência nos serviços de saúde, em razão da falta de infraestrutura e de profissionais de saúde para atender as necessidades da família. 0 estudo de Molina, Fonseca, Waidman \& Marcon (2009) realizou-se em Unidade de Terapia Intensiva e Neonatal. Nesse estudo, a família vivenciou momentos de tensão, mas considerou que a presença numa unidade de terapia intensiva possibilita auxiliar a recuperação da criança, e o acolhimento e a percepção de cuidados recebidos da equipe de saúde representaram aspectos positivos para a recuperação do filho.

A participação do acompanhante em um grupo de pais numa unidade neonatal foi analisada em um estudo (Ferreira, Sakita, \& Ceccon, 2009). Os relatos dos pais evidenciaram que a participação no grupo contribui para o esclarecimento de informações referentes à condição clínica da criança, o que repercute na tranquilidade, na segurança, no cuidado e nas interações com a criança. A pesquisa conclui que 0 grupo deve ser visto como um primeiro passo em direção ao melhor que cada profissional de saúde pode oferecer na assistência ao recém-nascido e sua família. O grupo de pais com finalidade de promover ações educativas mostra-se um recurso efetivo para estabelecer comunicação e compartilhar vivências que auxiliam os familiares no manejo das situações que envolvem o adoecimento de uma criança. 
Os significados do adoecimento e hospitalização de mães de crianças com câncer foram analisados (Hansson, Kjaergaard, Schmiegelow, \& Hallstrom, 2012). O estudo descreve as experiências de um programa de cuidados hospitalares em domicílio oferecido às famílias de crianças com câncer, e os resultados indicam que a atenção domiciliar de base hospitalar permitiu às famílias permanecerem tranquilas durante todo o curso do tratamento, uma vez que diminuiu a pressão sobre a família e a criança doente, e foi mantida uma vida cotidiana normal que cumpriu a necessidade de proteção e segurança.

No que se refere as reestruturações familiares diante do câncer de uma criança/adolescente, os autores identificaram que a figura materna vivencia sofrimento decorrente da tarefa de organização e manutenção do contexto familiar, o que inclui a coesão e a integridade de seus integrantes (Quintana, Wotrich, Camargo, Sherer, \& Ries, 2011). Essa responsabilização no manejo da crise, algumas vezes, gera culpa e desamparo, quando existem dificuldades para o manejo das situações. Os autores refletem sobre o papel dos profissionais de saúde na condição de facilitadores do processo de elaboração de lutos vivenciados pela família.

Alguns estudos analisaram as necessidades de mães durante a hospitalização da criança (Angelo, Moreira, \& Rodrigues, 2010; Franco \& Aguilar, 2007). As mães consideram importante acompanhar o filho hospitalizado, e as principais necessidades relacionavam à manutenção do vínculo e o recebimento de apoio da família. Os resultados revelaram que profissionais de saúde não têm mediado suas ações a partir de uma efetiva relação profissionalfamília-criança, entendendo que a humanização do cuidar perpassa pelo compartilhar de perspectivas (Franco \& Aguilar, 2007).

Uma pesquisa procurou analisar as vivências de familiares de crianças internadas no pronto-socorro (Santos et al., 2011). Durante o período de hospitalização, o acompanhante vivencia conflitos e adaptações provenientes das mudanças na rotina da família. Estes conflitos estão relacionados com as dificuldades para permanecer acompanhando a criança no hospital, ao considerar que a vigilância constante para promover os cuidados básicos e a distância da família ocasionam angústia e tristeza. Diante dessa situação, os acompanhantes utilizam como formas de enfrentamento o contato com os familiares e a utilização de práticas religiosas.

Procurou-se investigar os efeitos da preparação psicológica précirúrgica de crianças submetidas a dois modelos de intervenção (o primeiro relacionava a transmissão de informações verbais sobre a cirurgia, e o segundo a utilização de brinquedos para informar a criança), segundo as percepções das mães (Broering \& Crepaldi, 2011). Foram obtidos dados positivos que indicam a importância da preparação psicológica para crianças submetidas aos procedimentos 
cirúrgicos, independentemente do tipo de preparação, pois as mães perceberam que seus filhos apresentaram mudanças positivas no humor, redução de ansiedade, e as informações recebidas pelas crianças contribuíram para o esclarecimento de dúvidas. Verificou-se a relevância da preparação psicológica para crianças hospitalizadas, o que facilita a inserção da criança nos procedimentos realizados no ambiente hospitalar, e contribui para a qualidade do relacionamento entre mãe e filho.

\subsubsection{Estresse, enfrentamento e suporte familiar}

As pesquisas focalizam a avaliação dos níveis de estresse, as estratégias de enfrentamento, e o suporte familiar. Estudos sobre estresse dos pais e sintomas de estresse pós-traumático decorrentes do acompanhamento de crianças hospitalizadas, assim como as estratégias utilizadas para enfrentar as situações. Níveis de suporte familiar percebidos pelos pais são apresentados contextualizando a dinâmica da família. Uma das situações avaliadas foi à presença de estresse e a satisfação dos pais durante a hospitalização (FernándezCastillo, Vílchez-Lara, \& López-Naranjo, 2012). A ocorrência de estresse foi decorrente da situação de doença e hospitalização da criança, e das mudanças nas relações com a família e profissionais de saúde. Os homens apresentaram maiores níveis de satisfação geral com a internação, se comparados às mulheres, e assim, os autores sugerem que sejam realizadas mudanças nos cuidados que são oferecidos no hospital visando a satisfação geral dos pais.

Sintomas de estresse pós-traumático em familiares de crianças hospitalizadas foram investigados em dois estudos (Bruce, Gumley, Isham, Fearon, \& Phipps, 2011; Coakley et al., 2010), e os resultados indicaram a prevalência de sintomas que caracterizam sofrimento vivenciado após uma situação traumática. Os sintomas de estresse pós-traumático foram associados a outras variáveis, por exemplo, a resolução de conflitos nas relações entre pais e filhos, e as recidivas tumorais (Bruce et al., 2011). O funcionamento familiar apresentou uma associação com o desenvolvimento dos sintomas de estresse pós-traumático nos pais de crianças que sofreram uma lesão traumática acidental (Coakley et al., 2010). Os resultados permitem compreender que após o período de hospitalização da criança, os familiares acompanhantes necessitam de atenção integral para minimizar as repercussões psicológicas provenientes desta experiência. Existe a importância da avaliação psicológica para verificar níveis de estresse nestas situações, assim como o direcionamento de intervenções quando estes familiares se encontram no ambiente hospitalar.

Procurou-se investigar os sentimentos vivenciados e as estratégias de enfrentamento de mães que acompanhavam crianças hospitalizadas 
(Costa, Mombelli, \& Marcon, 2009). O medo e a tristeza provocaram o surgimento de ansiedade, e para enfrentar as situações, as mães procuravam estabelecer relações sociais e brincar com a criança, ou reagir por meio de ações isoladas como, por exemplo, chorar, ficar sozinha e utilizar práticas religiosas. Os autores sugerem que as equipes de saúde utilizem o acolhimento por meio de uma relação de confiança com a criança e família para auxiliar as mães nas vivências desses sentimentos e nas estratégias de enfrentamento.

A percepção do suporte familiar e saúde mental em mães de crianças hospitalizadas em unidade de terapia intensiva pediátrica representou objeto de investigação em diferentes fases: hospitalização, após a alta do setor, e um mês pós-hospitalização (Righetto-Dias \& NunesBaptista, 2009). Ocorreram variações na saúde mental, a melhora foi verificada na fase de alta hospitalar, e não houve variação na percepção do suporte familiar ao longo dos períodos de avaliação para esta amostra. O estudo indica que mães de crianças hospitalizadas apresentam alterações na saúde mental, e que necessitam de intervenção focalizada nestes aspectos para auxiliar 0 acompanhamento da criança.

\subsubsection{Família e equipe de saúde}

As pesquisas evidenciam aspectos referentes às interações entre a família da criança hospitalizada e equipe de saúde. Estudos sobre as relações estabelecidas pelas enfermeiras com a família durante a hospitalização infantil, os significados da internação hospitalar pediátrica, a comunicação equipe e família na unidade de terapia intensiva, e as percepções das famílias sobre os profissionais de saúde. Verificou-se três estudos sobre a relação da equipe de saúde com a criança e família no ambiente hospitalar, de maneira específica, os profissionais de enfermagem (Aein et al., 2011; Fegran, Fagermoen \& Helseth, 2008; Souza, Scatolin, Ferreira, \& Croti, 2008).

A construção de uma teoria da interação enfermeira-mãe foi o objetivo dos pesquisadores, e identificou-se que a mãe da criança hospitalizada procura estabelecer fronteiras por meio do enfrentamento das pressões ambientais, da conscientização dos deveres da enfermeira, e das relações estabelecidas com essas profissionais (Aein et al., 2011). O estudo de Fegran et al. (2008) apresenta uma análise da parceira de pais e enfermeiros em três momentos da doença: a fase aguda crítica, a fase de estabilização, e a fase de descarga. A fase de estabilização foi considerada desafiadora pelo fato de ocorrer o esgotamento físico e mental dos pais e, diante desta situação, os pais consideram importante estabelecer uma relação de confiança com os enfermeiros para auxiliar nas responsabilidades e adaptações, e os enfermeiros 
buscavam desenvolver a autonomia dos pais nos cuidados com a criança. Sobre a relação da equipe de enfermagem com a criança e a família em pós-operatório imediato de cardiopatias congênitas, os enfermeiros desenvolvem ações isoladas, por exemplo, a orientação acerca de procedimentos de rotina e evolução da criança (Souza et al., 2008), e os autores sugerem a necessidade da atenção integral as necessidades da criança e família.

Nas interações das enfermeiras com as mães de crianças hospitalizadas, verificou-se que existem problemas referentes à ausência do acolhimento e do manejo junto à criança e sua família (Lima, Silva, Collet, Reichert, \& Oliveira, 2010). O diálogo entre equipe de saúde e família da criança hospitalizada foi discutido pelos autores, 0 que permite evidenciar a importância das relações interpessoais desenvolvidas no ambiente hospitalar.

Os significados da internação hospitalar pediátrica na perspectiva de profissionais de enfermagem e familiares foram investigados (Mariano et al., 2011). Para estes participantes, o processo de hospitalização ocasiona mudanças nas esferas familiar e social mas, por outro lado, existem possibilidades de organização quando o suporte é oferecido, assim como a transmissão de informações educativas. Ao analisar as concepções da equipe de enfermagem acerca da mãe acompanhante (Quirino, Collet, \& Neves, 2010), os resultados indicam que estes profissionais reconhecem a importância da mãe no acompanhamento da criança hospitalizada, embora todas as decisões sobre 0 tratamento da criança sejam de responsabilidade da equipe de saúde. A comunicação equipe-família em unidade de terapia intensiva pediátrica representou o objeto de investigação, na perspectiva dos familiares, o que permitiu compreender que o processo de comunicação apresentava repercussões nas interações entre os sujeitos, de maneira específica na ocorrência de sofrimento emocional dos familiares, e de outro lado, nas ações profissionais realizadas pela equipe de saúde (Nieweglowski \& Moré, 2008). Um exemplo da dificuldade de comunicação foi identificado nos casos em que o diagnóstico da criança era imprevisível, e diante do agravamento da condição clínica. Nestas situações, os familiares vivenciavam ansiedade, e a equipe de saúde apresentava dificuldades em estabelecer o acolhimento e a manutenção do diálogo, pelo fato de que a situação gerava desconforto. Os autores indicam a necessidade de capacitação dos profissionais de saúde, no que se refere ao processo de comunicação com os familiares, para que os mesmos fiquem atentos durante a transmissão e compartilhamento de informações, para que seja possível auxiliar as relações que são desenvolvidas e minimizar as repercussões psicológicas vivenciados por todos os envolvidos.

No que se refere às percepções de pais sobre o suporte recebido pelas enfermeiras durante o período de hospitalização da criança (He, 
Vehviläinen-Julkunen, Pölkki, \& Pietilä, 2010), os familiares receberam informações necessárias sobre procedimentos cirúrgicos e - manejo no pós-operatório, o que contribuiu para o acompanhamento da criança. Entretanto, não receberam informações suficientes sobre medicação para dor, logo, os resultados sugerem que os pais precisam de mais informações relacionadas com o manejo da dor e deve ser assegurada a sua compreensão das informações. Pesquisa sobre as experiências de pais e enfermeiras durante os cuidados da criança hospitalizada (Aein, Alhani, Mohammadi, \& Kazemnejad, 2009), apresentou resultados que indicam a importância da colaboração entre pais e enfermeiras, considerando que os familiares cumprem um papel fundamental nos cuidados destinados a criança. Sobre as interações família e equipe de saúde (Monticelli \& Boehs, 2007), existe uma aproximação e distanciamento devido às regras do ambiente hospitalar, que de alguma forma dificultam a autonomia destes indivíduos, e por isso, alguns familiares são resistentes às instruções que são fornecidas, como, por exemplo, quando são solicitados para auxiliar na hora do banho da criança e questionam o procedimento. Nas situações em que o familiar se encontra estressado, a equipe apresenta reações de medo, de fuga, ou solicita apoio da enfermeira.

\subsection{Família e adulto hospitalizado}

Os estudos em torno da família do adulto hospitalizado integram temáticas referentes à experiência da família, estresse e enfrentamento, e a relação família e equipe de saúde.

\subsubsection{Experiência da família}

As pesquisas apresentam resultados referentes às experiências de famílias diante da hospitalização de um adulto: experiência da família de um adulto hospitalizado com doença crítica, sentimentos de familiares de pacientes hospitalizados, concepções acerca da hospitalização, e necessidades e vivências de famílias de pacientes hospitalizados na Unidade de Terapia Intensiva (UTI). A análise da experiência da família de um adulto hospitalizado com doença crítica permitiu identificar que as vivências ocorrem de maneira processual e envolvem etapas por meio de um ciclo que possibilita o desenvolvimento de habilidades para enfrentar o período de hospitalização (Eggenberger \& Nelms, 2007). Para os autores, o manejo desta experiência possibilita o diálogo entre os familiares e provoca um efeito terapêutico, o que permite facilitar as relações estabelecidas no ambiente hospitalar. A hospitalização representou para os familiares um momento crítico marcado pelo medo, e por mudanças na rotina de vida com repercussões para a dinâmica 
familiar (Sousa-Filho, Xavier, \& Vieira, 2008). As motivações de familiares para acompanhar os pacientes hospitalizados estão relacionadas com o apoio para garantir os cuidados necessários, assim como foi possível verificar que preocupações referentes à evolução clínica contribuem para permanecer o maior número de horas no hospital (Auslander, 2011). De acordo com estas pesquisas, é possível compreender que na situação de doença e hospitalização, as famílias de pacientes adultos vivenciam sofrimento psicológico e mudanças na rotina de vida. Entretanto, o apoio destinado ao paciente representa um recurso que auxilia 0 processo de recuperação.

As vivências de famílias de adultos hospitalizados com doença crônica foram analisadas em dois estudos (Miliorini, Fernandes, Decesaro, \& Marcon, 2008; Schneider, Manschein, Ausen, Martins, \& Albuquerque, 2008), e de forma específica a implantação da tecnologia leve de cuidado denominada de acolhimento (Schneider et al., 2008). Os anseios e expectativas da família relacionados à doença crônica integram, inicialmente, sentimento de tristeza e a utilização de práticas religiosas para enfrentar as situações, ao considerar as possibilidades de recuperação e 0 apoio oferecido ao paciente (Miliorini et al. 2008). No estudo de Schneider et al. (2008), a ocorrência do infarto e a necessidade de hospitalização foram associados pelo paciente e a família às percepções de morte e sentimentos de pânico, de forma que o acolhimento foi considerado o apoio que permite atender às suas necessidades. Baseando-se na pesquisa, os autores elaboraram uma proposta de acolhimento por meio de etapas (acesso, escuta, diálogo, apoio e vínculo), e de ações específicas a serem realizadas pela equipe de saúde.

A investigação sobre os sentimentos de familiares de pacientes hospitalizados (Beuter, Brondani, Szareski, Cordeiro, \& Roso, 2012), possibilitou destacar a solidão, o abandono, e o desespero destes familiares, aspectos que ocasionaram sofrimento psíquico. Familiares de pacientes hospitalizados com queimaduras vivenciam uma fase de crise que repercute nos aspectos físicos e emocionais, no relacionamento e rotina, e estes aspectos, de maneira geral, modificam a dinâmica familiar, e diante desta situação, ocorre um ciclo de crise, adaptação e estabilização (Carvalho, Rossi, \& CiofiSilva, 2008).

A análise do funcionamento dos membros da família do paciente hospitalizado mediante a ocorrência de um coma traumático, evidenciou que estes familiares vivenciam experiências por meio de fases ou ciclos: 1. A busca da proteção de sua vida e de seus parentes, e 2. A reconstrução de suas experiências e de seus parentes (Verhaeghe, Van Zuuren, Grypdonck, Duijnstee, \& Defloor, 2010). Essa busca da proteção da vida foi referida a uma batalha, na qual diariamente busca-se enfrentar os acontecimentos da vida para 
garantir a integridade da família, e que gradualmente isso possibilita reconstruir as experiências que são vivenciadas.

Três estudos analisaram as vivências de familiares na sala de espera da UTI (Frizon, Nascimento, Bertoncello, \& Martins, 2011; Obringer, Hilgenberg, \& Booker, 2012; Urizzi \& Corrêa, 2007). Sentimentos relacionados à tristeza, medo e desespero associados com a expectativa de alta hospitalar (Frizon et al., 2011); necessidades de garantias a serem atendidas na unidade hospitalar (Obringer et al., 2012); e a experiência de acompanhar um familiar foi considerada difícil devido as mudanças no ambiente familiar e as preocupações com os cuidados, aspectos que geram sofrimento, pelo fato de que ambos compartilham as experiências no contexto da hospitalização.

Familiares consideram importante que a equipe de saúde apresente informações sobre a evolução clínica de pacientes hospitalizados, e isto representa a principal necessidade destes familiares (Lucchese, Citero, Marco, Andreoli, \& Nogueira-Martins, 2008). Diante destes achados, torna-se possível ressaltar que as famílias necessitam de informações sobre o quadro clínico do paciente e consideram importante o acolhimento recebido pela equipe de saúde, fatores que permitem minimizar as repercussões psicológicas provenientes da vivência de hospitalização.

\subsubsection{Estresse e enfrentamento}

Familiares de pacientes hospitalizados apresentam altos níveis de estresse (Leff et al., 2007), e nessa amostra, as mulheres apresentaram os maiores indicadores, se comparado aos homens, 0 que permitiu indicar que existe uma correlação entre a ocorrência de estresse e a utilização de estratégias de enfrentamento (Chui \& Chan, 2007). Estes estudos indicam que a hospitalização de um membro da família ocasiona o surgimento de estresse nos seus familiares, e a utilização das estratégias de enfrentamento permite facilitar 0 desenvolvimento de habilidades. Verificou-se que as estratégias de enfrentamento de familiares apresentaram variações, sendo a mais frequente o suporte social (Bomfim, Bastos, \& Carvalho, 2007). Assim, o contato com as pessoas próximas representa o principal recurso utilizado para garantir apoio e enfrentar o período vivenciado no ambiente hospitalar.

\subsubsection{Família e equipe de saúde}

Pesquisas cujos conteúdos abordam as percepções da equipe de enfermagem sobre a família do paciente hospitalizado, e o processo de comunicação entre a equipe de enfermagem e famílias de pacientes no contexto hospitalar. As percepções da equipe de enfermagem sobre o familiar acompanhante no cuidado ao adulto 
hospitalizado relacionam a importância da presença do familiar, as funções desenvolvidas no ambiente hospitalar, e a necessidade de cuidados básicos para facilitar a recuperação do paciente, por exemplo, quando o familiar oferece água ao paciente e fica atento às alterações comportamentais (Szareski, Beuter, \& Brondani, 2010). Nos casos em que o familiar participa dos cuidados, a equipe considera um aspecto positivo, mas quando o familiar não se mostra disponível, a sua presença gera conflitos na relação com a equipe (Szareski et al., 2010). Numa pesquisa sobre o perfil de familiares acompanhantes de pacientes hospitalizados com doenças crônicas para verificar as possibilidades de ações educativas da equipe de enfermagem, verificou-se a prevalência do gênero feminino, constituída de esposas, mães e filhas, com baixo nível de escolaridade (Beuter, Brondani, Szareski, Lana, \& Alvim, 2009). Os autores destacam que a identificação do perfil socioeconômico do familiar acompanhante permite que a equipe de enfermagem direcione estratégias de educação à saúde por meio de medidas de suporte à família, para que estes indivíduos possam utilizar diferentes formas de enfrentamento.

A análise da comunicação entre os profissionais de enfermagem e familiares de pacientes hospitalizados na unidade de terapia intensiva (Silva, Prochnow, Santos, Guerra, \& Barrios, 2009) indicou que os familiares necessitam de informações, por meio de uma linguagem de fácil compreensão, a respeito da condição clínica do paciente e dos procedimentos que estão sendo realizados. Os autores apresentam reflexões acerca do acolhimento no ambiente hospitalar, o que representa uma estratégia básica para atender às demandas dos familiares e auxiliar no apoio. Percebe-se que os problemas relativos à falta de informações se reflete nas dificuldades de comunicação entre familiares e equipe de enfermagem, de maneira específica na satisfação com o atendimento da equipe, e, portanto, ações que envolvam equipes de saúde e familiares são consideradas emergentes para o manejo desta situação.

\subsection{Família e idoso hospitalizado}

Os estudos sobre família e idoso hospitalizado integram temáticas acerca da importância dos familiares e a relação entre família e equipe de saúde.

\subsubsection{A relevância da família}

Um estudo de caso clínico analisou as percepções de um familiar acompanhante de idoso hospitalizado na iminência da morte (Perez, Silva, \& Couto, 2009), foi possível verificar que existem fases de enfrentamento da morte que são vivenciadas pelos familiares, o que 
ocorre por meio de um ciclo que gradualmente permite a elaboração desta experiência. Na investigação sobre os fatores que impediram os familiares de ficarem na condição de acompanhantes do idoso no ambiente hospitalar (Souza, 2011), verificou-se que os familiares estavam vivenciando uma fase de organização do funcionamento familiar, o que apresentou repercussões no grupo familiar que impossibilitou a presença permanente no hospital. Os resultados permitem compreender as mudanças provocadas nas famílias quando um parente se encontra hospitalizado, principalmente nas situações de terminalidade e morte, que exigem atenção e cuidados especiais.

Um programa de intervenção para familiares cuidadores de pacientes idosos hospitalizados foi testado em dois grupos de intervenção e controle (Li et al., 2012). As intervenções foram realizadas em duas sessões em momentos distintos: o primeiro grupo participou no período de 1-2 dias pós-admissão, e 1-3 dias na fase pré-alta, o que ocorreu por meio da transmissão de informações educacionais referentes às características do ambiente hospitalar e dos cuidados necessários para o paciente hospitalizado. O grupo controle recebeu informações genéricas sobre procedimentos hospitalares em duas sessões no mesmo período de tempo do grupo de intervenção. Foram aplicados instrumentos psicométricos para avaliar depressão, ansiedade e crenças. Ocorreu um seguimento das amostras no período de duas semanas e dois meses pós-hospitalização. Os resultados indicaram que o segundo grupo apresentou escores elevados nos indicadores de ansiedade e depressão, se comparado ao primeiro grupo que obteve benefícios com a intervenção. De acordo com Li et al. (2012), as evidências pouco consistentes dos resultados da pesquisa mostram a importância das ações educativas no ambiente hospitalar destinadas aos familiares de pacientes hospitalizados, e considera que as orientações permitem evitar o surgimento de sintomas de ansiedade e depressão.

\subsubsection{Família e equipe de saúde}

Nesta subcategoria são apresentados estudos sobre as expectativas da equipe de enfermagem referentes às atividades realizadas pelos familiares de idosos hospitalizados, e as relações entre apoio familiar e o processo de trabalho em enfermagem. Na busca pela investigação das atividades realizadas pelos familiares cuidadores no acompanhamento ao idoso hospitalizado, assim como quais atividades a equipe de enfermagem espera que sejam realizadas pelo cuidador (Pena \& Diogo, 2009), foram identificados os cuidados básicos relacionados à higiene pessoal, e atividades que facilitam a recuperação do paciente hospitalizado, por exemplo, auxiliar durante as refeições. 
Na pesquisa sobre o papel do enfermeiro nas rotinas hospitalares relativas aos familiares de idosos hospitalizados (Bocchi, Silva, Juliani, \& Spirit, 2007), os resultados indicaram que os enfermeiros apresentam papel importante no apoio às famílias, e foram apresentadas reflexões críticas acerca das regras estabelecidas pela instituição hospitalar que dificultam a autonomia dos profissionais e a integração do familiar na equipe de saúde. Um estudo investigou as perspectivas de pacientes e seus familiares sobre o papel das enfermeiras durante o processo de reabilitação na unidade hospitalar (Tyrrell, Levack, Ritchie, \& Keeling, 2012). Foram realizadas entrevistas com sete pacientes e seis membros familiares, e utilizouse a teoria fundamentada nos dados para análise dos relatos. Os familiares reconhecem a importância da enfermagem, e a relação desenvolvida por estes profissionais foi considerada positiva para facilitar a participação dos pacientes na reabilitação, e assim, desenvolver autonomia e motivação para a continuidade do tratamento. A investigação das relações desenvolvidas entre profissionais de saúde e membros de familiares idosos hospitalizados apresenta relevância devido à identificação de fatores que permitem aprimorar a atuação da equipe, no que se refere ao acolhimento destinado aos familiares.

\section{Discussão}

Nas investigações realizadas com familiares de crianças hospitalizadas, provavelmente 0 fator responsável pelo número expressivo de publicações é decorrente da sensibilização dos efeitos psicológicos provocados na criança hospitalizada e família, o que impulsionou profissionais de saúde e pesquisadores no desenvolvimento de estudos para o aprofundamento da temática. A consolidação de áreas específicas de pesquisas, a exemplo da Psicologia Pediátrica no contexto nacional (Crepaldi, Rabuske \& Gabarra, 2006) e internacional (Barros, 2003; Roberts \& Steele, 2009) contribuiu para os avanços nas investigações, no aprimoramento de estudos teóricos, e na formação de pesquisadores. Os respectivos autores identificaram que as reações psicológicas vivenciadas pela criança hospitalizada, a saber, o medo, a insegurança, e as oscilações no humor e comportamento representam aspectos compartilhados pelo familiar cuidador, de maneira que a relação de empatia que é desenvolvida repercute no sofrimento psicológico da díade (criança/cuidador). É provável que as ações políticas para garantir atenção à criança hospitalizada e aos seus familiares foram desenvolvidas buscando minimizar as repercussões psicológicas. 
A Lei 8.069/90 do Estatuto da Criança e do Adolescente (ECA) assegurou 0 direito de a criança hospitalizada receber acompanhamento de um familiar em período integral, para que seja possível auxiliar a proteção e manutenção da saúde da criança (Brasil, 1990). No período anterior à construção desta lei, os profissionais de saúde, especificamente os enfermeiros e psicólogos, sensibilizados com o sofrimento da criança hospitalizada buscavam desenvolver o auxílio no decorrer de suas práticas de cuidado, o que possibilitou o desenvolvimento de estudos teóricos e empíricos. Desta forma, isso explica o fato de que os estudos sobre família e criança hospitalizada estão concentrados na área da enfermagem, o que foi verificado nessa revisão sistemática. Estes profissionais construíram uma área de pesquisa, e que logo foi compartilhada por outras áreas, a exemplo da Psicologia, que atualmente apresenta uma área de pesquisa denominada de Psicologia da Saúde da Criança e do Adolescente. A contribuição da Psicologia nos estudos sobre família e criança hospitalizada tem sido enfatizada por meio de publicações que apresentam resultados de pesquisas empíricas, o que auxilia na formulação de estudos teóricos (Barros, 2003; Crepaldi, Rabuske \& Gabarra, 2006; Roberts \& Steele, 2009).

No que se refere à produção científica de família e criança hospitalizada, as vivências, a avaliação de aspectos psicológicos, e as relações entre família e equipe de saúde foram as categorias extraídas de pesquisas. Os estudos continuam sendo desenvolvidos buscando a exploração das experiências de familiares, ao considerar que nos relatos foram identificadas as dificuldades provenientes da distância da família, o desgaste físico e emocional do cuidador, o enfrentamento de novas rotinas e a necessidade de um espaço para expressar pensamentos e compartilhar as vivências com outras pessoas do ambiente hospitalar (Santos, Oliveira, Barbosa, Siqueira \& Peixoto, 2013). No contexto de cuidados intensivos, os vínculos afetivos representam o principal ponto para os familiares permanecerem ao lado da criança, mas existe uma necessidade de solicitar informações referentes à condição clínica para acompanhar o processo de recuperação (Cardoso, Rodrigues, Pacheco, \& Araújo, 2013).

Novos estudos foram desenvolvidos com o interesse de avaliação de práticas e mensuração da ansiedade do familiar por meio de pesquisa experimental (Sampaio, Silva, Comino, \& Romano, 2014). Foram construídos dois grupos de familiares, o primeiro recebia informações da enfermagem referentes à cirurgia a que a criança seria submetida, e o segundo grupo não recebia informações, sendo possível verificar que o primeiro grupo apresentou baixos indicadores de ansiedade. Em outro estudo que analisou as opiniões de mães de bebês hospitalizados sobre as intervenções da enfermagem, identificou-se que as ações da enfermagem (conversar com a mãe sobre as 
características do temperamento do bebê/ oferecer oportunidade para a mãe segurar o bebê e realizar os cuidados/ promover a participação da mãe nas decisões sobre os cuidados do filho) foram avaliadas de maneira positiva, e segundo as mães isso possibilitou 0 desenvolvimento da função materna e o desempenho de cuidados destinados à criança (Neiva, Guimarães, Vale, \& Carmona, 2013). Nesses estudos, é possível verificar que as ações da enfermagem na área da pediatria estão sendo avaliadas, 0 que permite 0 aprimoramento das práticas para atender às necessidades da criança e família.

A temática do cuidado também tem sido investigada, no que se refere às percepções do enfermeiro sobre a inserção do familiar no cuidado destinado a criança, e se verificou uma percepção positiva e a importância atribuída ao contato entre profissional de saúde e familiar cuidador, buscando fortalecer vínculos (Facio, Matsuda, \& Higarashi, 2013). Outros estudos enfatizaram as relações entre o trabalho do enfermeiro e o processo de cuidado à criança por parte dos familiares (Serrano \& Larrayad, 2014; Silva, Santos, Rocha, Valença, \& Bay-Júnior, 2015), e o que se verifica nesses estudos é uma valorização da presença do familiar cuidador. É possível compreender que nas relações entre equipe de saúde e familiar cuidador prevalecem as investigações sobre a temática do cuidado, 0 que apresenta congruência com os resultados de uma revisão integrativa sobre interação equipe de enfermagem, família e criança hospitalizada (Azevêdo, Lançoni-Júnior, \& Crepaldi, 2016). Esses avanços são importantes para aprimorar os estudos científicos e desenvolver reflexões sobre estratégias de intervenção e pesquisa. Isso permite auxiliar a equipe de saúde na relação com o familiar cuidador por meio da humanização nas práticas em saúde.

Em relação à família do adulto hospitalizado, a prevalência de estudos qualitativos é decorrente da necessidade de compreender a maneira pela qual o sistema familiar vivencia o processo de adoecimento e hospitalização. Nessa revisão sistemática, a experiência da família foi investigada e os estudos encontraram pontos em comum, a exemplo do envolvimento do grupo familiar na organização das experiências e na elaboração de situações consideradas traumáticas. Os estudos atuais têm direcionado as investigações para a compreensão da experiência da família (Mistura, Schenkel, Rosa, \& Girardon-Perlini, 2014; Passos, Pereira, \& Nietsche, 2015; Vidal, Araújo, Perreault, \& Azevedo, 2013), e os resultados são semelhantes se comparados às pesquisas realizadas anteriormente. Os familiares cuidadores destacam a reorganização do funcionamento familiar para a realização das tarefas do ambiente doméstico, o desgaste físico decorrente da infraestrutura do ambiente hospitalar que interfere na qualidade do sono, as dificuldades de comunicação com os médicos, os sentimentos de tristeza, e a busca de apoio da família para 
enfrentar as situações (Mistura et al., 2014); o distanciamento da família e das atividades profissionais para ficar ao lado do paciente, as rotinas da instituição hospitalar, e o desgaste emocional, ao considerar que as alterações vivenciadas repercutem nas relações com as pessoas da família (filhos, cônjuge), no trabalho, e no cuidado à saúde (Passos et al., 2015).

De maneira específica, um estudo buscou identificar as reações do paciente antes, durante e depois da permanência do familiar cuidador na unidade de terapia intensiva, e os resultados indicaram que ocorreram mudanças verbais e não-verbais, a exemplo das variações na fala, movimentos, adesão ao tratamento, e melhorias na comunicação e na participação do cuidado (Vidal et al., 2013). O que se verifica é a utilização de delineamento experimental, o que contribui para a ampliação das possibilidades de pesquisa e intervenção.

Nas pesquisas sobre família e idoso hospitalizado, as quais apresentaram menor frequência, os estudos buscaram investigar de maneira qualitativa a experiência da família e as relações entre família e equipe de saúde. As pesquisas atuais seguem essa tendência (Chernicharo \& Ferreira, 2015; Chibante, Espírito-Santo, \& Aquino, 2015), embora seja possível identificar uma delimitação do objeto de estudo, a exemplo do estresse, e o aprimoramento de estudo da temática referente ao cuidado. Os autores identificaram que as mudanças vivenciadas pelos familiares relacionam as alterações no humor, alimentação, e uso de psicotrópicos, e para o enfrentamento do estresse, os familiares buscam desenvolver atividades de lazer e a utilização de práticas religiosas (Chibante et al., 2015). Em relação aos sentidos atribuídos ao cuidado, os familiares relacionam ao auxílio que é oferecido, a relação entre familiar, paciente e profissional de saúde enfermeiro, ao apoio institucional e as orientações de cuidado (Chernicharo \& Ferreira, 2015). Em síntese, existem poucas publicações sobre família e idoso hospitalizado, o que representa uma área para exploração. Assim, recomenda-se a realização de estudos para que os resultados possam aprimorar práticas de atenção à saúde integral. De maneira geral, os estudos sobre família e criança hospitalizada continuam em expansão, e em seguida àqueles referentes ao familiar do adulto, no âmbito nacional e internacional, e verificou-se que no Brasil os pesquisadores utilizam o método qualitativo. Assim, recomenda-se que os pesquisadores reflitam sobre os resultados de pesquisas para o desenvolvimento de aplicações práticas que contribuam para a integralidade da assistência ao familiar cuidador que se encontra no contexto hospitalar. 


\section{Considerações finais}

Na produção científica de artigos sobre a família no contexto da hospitalização, verificou-se maior número de pesquisas sobre família e criança hospitalizada, o que permite destacar o crescimento de publicações nacionais e internacionais, principalmente nos periódicos da área da enfermagem. No que se refere à categoria família e adulto hospitalizado, foram encontrados poucos estudos publicados, os dados relevantes apontam o sofrimento vivenciado pelas famílias, as repercussões psicológicas provenientes desta experiência, e a necessidade de estabelecimento da comunicação com a equipe de saúde. Um ponto específico refere-se ao número limitado de pesquisas sobre família e idoso hospitalizado, considerando que foram identificados os artigos publicados nos últimos seis anos (2007, 2008, 2009, 2010, 2011, 2012).

De maneira geral, nas três categorias verificou-se que a família vivencia uma fase de impacto, que se refere a um choque, quando um parente próximo se encontra hospitalizado, e as reações psicológicas são potencializadas quando o indivíduo está hospitalizado no ambiente de cuidados intensivos. Para a comunidade científica, sugere-se que os pesquisadores focalizem a atenção para os aspectos relacionados à saúde deste familiar numa perspectiva da integralidade, com ações de promoção e proteção das condições saudáveis. Na literatura pesquisada, não foi possível identificar programas de intervenção para minimizar o estresse e potencializar o enfrentamento. A avaliação do sofrimento psíquico e a utilização de intervenções representam estratégias úteis a serem utilizadas no ambiente hospitalar, para que estes familiares possam facilitar 0 manejo nas situações de adoecimento. Outros recursos importantes a serem utilizados pelas equipes de saúde para preservar a saúde do familiar que acompanha um paciente hospitalizado: acolhimento por meio da escuta qualificada e de uma relação empática, atenção às necessidades e demandas de cada familiar, abertura para o diálogo e transmissão de informações úteis, e inclusão do familiar nos grupos terapêuticos.

Os resultados indicam a importância atribuída pelos familiares de pacientes hospitalizados à comunicação estabelecida com a equipe de saúde, o que representa um ponto para ser considerado pelos profissionais que atuam no ambiente hospitalar. O objetivo da presente pesquisa foi atingido, considerando que o mapeamento de pesquisas sobre famílias no contexto de hospitalização permitiu compreender os estudos e as perspectivas para futuras investigações científicas na área da Psicologia. Recomenda-se que sejam realizadas pesquisas sobre a família do paciente adulto e idoso hospitalizado, para contribuir com o desenvolvimento da produção científica. 


\section{Referências}

Aein, F., Alhani, F., Mohammadi, E., \& Kazemnejad, A. (2009). Parental participation and mismanagement: a qualitative study of child care in Iran. Nursing \& health sciences, 11(3), 221-227.

Aein, F., Alhani, F., Mohammadi, E., \& Kazemnejad, A. (2011). Struggling to create new boundaries: a grounded theory study of collaboration between nurses and parents in the care process in Iran. Journal of advanced nursing, 67(4), 841-853.

Angelo, M., Moreira, P. L., \& Rodrigues, L. M. A. (2010). Incertezas diante do câncer infantil: compreendendo as necessidades da mãe. Escola Anna Nery Revista de Enfermagem, 14(2), 301308.

Arruda, D. C. D., \& Marcon, S. S. (2007). A família em expansão: experienciando intercorrências na gestação e no parto do bebê prematuro com muito baixo peso. Texto Contexto Enfermagem, 16(1), 120-128.

Auslander, G. K. (2011). Family caregivers of hospitalized adults in Israel: a point-prevalence survey and exploration of tasks and motives. Research in nursing \& health, 34(3), 204-217.

Azevêdo, A. V. S. A., Lançoni-Júnior, A. C., \& Crepaldi, M. A. (2016). Interação equipe de enfermagem, família e criança hospitalizada. Ciência \& Saúde Coletiva, 21(5), 28-39.

Barros, L. (2003). Psicologia pediátrica: perspectiva desenvolvimentista. Lisboa: Climepsi.

Bardin, L. (1979). Análise de conteúdo. Lisboa: Edições 70.

Beuter, M., Brondani, M., Szareski, C. M., Lana, L. D., \& Alvim, N. A. T. (2009). Perfil de familiares acompanhantes contribuições para a ação educativa da enfermagem. Revista Mineira de Enfermagem, 13(1), 28-33.

Beuter, M., Brondani, C. M., Szareski, C., Cordeiro, F. R., \& Roso, C. C. (2012). Sentimentos de familiares acompanhantes de adultos face ao processo de hospitalização. Escola Anna Nery Revista de Enfermagem, 16(1), 134-140.

Bomfim, A. C., Bastos, A. C., \& Carvalho, A. M. A. (2007). A família em situações disruptivas provocadas por hospitalização. Revista Brasileira Crescimento Desenvolvimento Humano, 17(1), 84-94.

Bocchi, S. C. M., Silva, L., Juliana, C. M. C. M., \& Spirit, W. Z. (2007). Familiares visitantes e acompanhantes de adultos e idosos hospitalizados: análise da experiência na perspectiva do processo de trabalho em enfermagem. Revista LatinoAmericana de Enfermagem, 15(2), 304-310.

Böing, E., Crepaldi, M. A., Moré, C. L. O. O. (2008). Pesquisa com famílias: aspectos teórico-metodológicos. Paidéia, 18(40), 251266.

Brasil (1990). Estatuto da Criança e do Adolescente. Lei n. 8.069, de 
13 de junho de 1990. Dispõe sobre o Estatuto da Criança e do Adolescente, e dá outras providências. Ministério da Ação Social (BR). Brasília (DF): Diário Oficial da União.

Bruce, M., Gumley, D., Isham, L., Fearon, P., \& Phipps, K. (2011). Post-traumatic stress symptoms in childhood brain tumour survivors and their parents. Child: care, health and development, 37(2), 244-251.

Broering, C. V., \& Crepaldi, M. A. (2011). Percepções das mães sobre a preparação pré- cirúrgica de seus filhos segundo dois modelos. Psicologia Argumento, 29(66), 327-341.

Cardoso, J. M. R. M., Rodrigues, B. M. R. D., Pacheco, S. T. A., \& Araújo, B. B. M. (2013). Ação intencional do familiar junto da criança em centro de terapia intensiva pediátrico. Revista de Enfermagem UERJ, 21(1), 600-605.

Carvalho, F. L., Rossi, L. A., \& Ciofi-Silva, C. L. (2008). A queimadura e a experiência do familiar frente ao processo de hospitalização. Revista Gaúcha de Enfermagem, 29(2), 199-206.

Coakley, R. M., Forbes, P. W., Kelley, S. D., Lebovidge, J., Beasley, P., Demaso, D. R., \& Waber, D. P. (2010). Family Functioning and Posttraumatic Stress Symptoms in Youth and Their Parents After Unintentional Pediatric Injury. Journal of Traumatic Stress, 23(6), 807-810.

Costa, J. B., Mombelli, M. A., \& Marcon, S. S. (2009). Avaliação do sofrimento psíquico da mãe acompanhante em alojamento conjunto pediátrico. Estudos de Psicologia, Campinas, 26(3), 317-325.

Crepaldi, M. A., \& Varella, P. B. (2000). A recepção da família na hospitalização de crianças. Paidéia (Ribeirão Preto), 12(1), 2333.

Crepaldi, M. A., Rabuske, M. M., \& Gabarra, L. M. (2006). Modalidades de atuação do psicólogo em psicologia pediátrica. In: M. A. Crepaldi, M. B. M. Linhares, \& G. B. Perosa (Orgs.), Temas em Psicologia Pediátrica (pp. 13-55). São Paulo: Casa do Psicólogo.

Chernicharo, I. M., \& Ferreira, M. A. (2015). Sentidos do cuidado com o idoso hospitalizado na perspectiva dos acompanhantes. Escola Anna Nery Revista de Enfermagem, 19(1), 80-85.

Chibante, C. L. P., Espírito Santo, F. H., \& Aquino, A. C. O. (2015). As reações do familiar acompanhante de idosos hospitalizados frente às situações de estresse. Revista Pesquisa: cuidado é fundamental, 7(3), 2961-2973.

Chui, W. Y. Y., \& Chan, S. W. C. (2007). Stress and coping of Hong Kong Chinese family members during a critical illness. Journal of clinical nursing, 16(2), 372-81.

Dessen, M. A., \& Braz, M. P. (2005). A família e suas inter-relações com o desenvolvimento humano. In: M. A. Dessen, \& A. L. 
Costa Júnior (Orgs.), A ciência do desenvolvimento humano: tendências atuais e perspectivas futuras (pp. 113-131). Porto Alegre: Artmed.

Dresch, A., Lacerda, D. P., \& Antunes Júnior, J. A. V. (2015). Método de pesquisa para avanço da Ciência e Tecnologia. Porto Alegre: Artmed.

Eggenberger, S. K., \& Nelms, T. P. (2007). Being family: the family experience when an adult member is hospitalized with a critical illness. Journal of clinical nursing, 16(9), 1618-1628.

Facio, B. C., Matsuda, L. M., \& Higarashi, I. H. (2013). Internação conjunta pediátrica: compreendendo a negociação enfermeiroacompanhante. Revista Eletrônica de Enfermagem, 15(2), 447453.

Lucchese, A. C., Citero, V. A., Marco, M. A., Andreoli, S. B., \& Nogueira-Martins, L. A. (2008). The needs of members of the families. São Paulo Medical Journal, 126(2), 128-131.

Fegran, L., Fagermoen, M. S., \& Helseth, S. (2008). Development of parent-nurse relationships in neonatal intensive care units from closeness to detachment. Journal of advanced nursing, 64(4), 363-371.

Fernández-Castillo, A., Vílchez-Lara, M. J., \& López-Naranjo, I. (2012). Parental Stress and Satisfaction during Children's Hospitalization: Differences between Immigrant and Autochthonous Population. Stress and health: journal of the International Society for the Investigation of Stress, 29(1), 2230.

Ferreira, J. C. O. A., Sakita, N. K., \& Ceccon, M. E. J. R. (2009). Experiência de grupo de pais em uma Unidade de terapia intensiva neonatal. Pediatria (São Paulo), 31(1), 20-25.

Franco, S. M. C. M., \& Aguilar, O. M. (2007). Compreendendo as necessidades de mães durante a hospitalização da criança. Pediatria, 43(2), 78-83.

Silva, J. L., Santos, E. G. O., Rocha, C. C. T., Valença, C. N., \& Bay Júnior, O. G. (2015). Organização do trabalho de enfermagem diante da inserção dos cuidados familiares com a criança hospitalizada. Revista Rene, 16(2), 226-233.

Sousa-Filho, O. A., Xavier, E. P., \& Vieira, L. J. E. S. (2008). Hospitalização na óptica do acidentado de trânsito e de seu familiar-acompanhante. Revista da Escola de Enfermagem da USP, 42(3), 539-546.

Frizon, G., Nascimento, E. R. P., Bertoncello, K. C. G., \& Martins, J. J. (2011). Familiares na sala de espera de uma unidade de terapia intensiva: sentimentos revelados. Revista Gaúcha de Enfermagem, 32(1), 72-78.

Hansson, H., Kjaergaard, H., Schmiegelow, K., \& Hallström, I. (2012). Hospital-based home care for children with cancer: a 
qualitative exploration of family members' experiences in Denmark. European journal of cancer care, 21(1), 59-66.

He, H. G., Vehviläinen-Julkunen, K., Pölkki, T., \& Pietilä, A. M. (2010). Chinese parents perception of support received and recommendations regarding children's postoperative pain management. International journal of nursing practice, 16(3), 254-61.

Leff, B., Burton, L., Mader, S. L., Naughton, B., Burl, J., Koehn, D., Clark, R., Greenough, W. B., Guido, S., Steinwachs, D., \& Burton, J. R. (2008). Comparison of stress experienced by family members of patients treated in hospital at home with that of those receiving traditional acute hospital care. Journal of the American Geriatrics Society, 56(1), 117-23.

Lima, A. S., Silva, V. K. B., Collet, N., Reichert, A. P. S., \& Oliveira, B. R. G. (2010). Relações estabelecidas pelas enfermeiras com a família durante a hospitalização infantil. Texto Contexto Enfermagem, 19(4), 700-708.

Li, H., Powers, B. A., Melnyk, B. M., McCann, R., Koulouglioti, C., Anson, E., Smith, J. A., Xia, Y., Glose, S., \& Tu, X. (2012). Randomized controlled trial of care: na intervention to improve outcomes of hospitalized elders and family caregivers. Research in Nursing \& Health, 35(5), 533-549.

Mariano, L. R. A., Backes, D. S., Ilha, S., Nicola, G. D. O., Freitas, H. M. B., \& Ferreira, C. L. L. (2011). Significados da internação hospitalar pediátrica na perspectiva de profissionais e familiares. Cogitare Enfermagem, 16(3), 511-516.

Serrano, A. M., \& Larrayad, M. E. (2014). Opinión del equipo de Enfermería de cuidados intensivos pediátricos sobre la participación de familiares en el cuidado. Metas enfermagem, 17(4), 6-11.

Minuchin, S. (1990). Famílias: funcionamento e tratamento. Porto Alegre: Artes Médicas.

Mistura, C., Schenkel, F. W., Rosa, B. V. C., \& Girardon-Perlini, N. M. O. (2014). A experiência em acompanhar um membro da família internado por câncer. Revista Pesquisa: cuidado é fundamental, 6(1), 47-61.

Monticelli, M., \& Boehs, A. E. (2007). A família na unidade de internação hospitalar: entre o informal e o instituído. Revista Escola Enfermagem da USP, 41(3), 468-477.

Molina, R. C. M., Fonseca, E. L., Angélica, M., Waidman, P., \& Marcon, S. S. (2009). A percepção da família sobre sua presença em uma Unidade de Terapia Intensiva Pediátrica e Neonatal. Revista latino-americana de enfermagem, 4(3), 630638.

Neiva, C. A. C., Guimarães, K. O., Vale, I. N., \& Carmona, E. V. (2013). Opinion of mothers of hospitalized babies about nursing 
interventions: a descriptive study. Online Brazilian Journal Nursing, 12 (4): 844-53.

Nieweglowski, V. H., \& More, C. L. O. O. (2008). Comunicação equipe-família em unidade de terapia intensiva pediátrica: impacto no processo de hospitalização. Estudos de Psicologia, Campinas, 25(1), 111-122.

Obringer, K., Hilgenberg, C., \& Booker, K. (2012). Needs of adult family members of intensive care unit patients. Journal of clinical nursing, 21(11-12), 1651-1658.

Miliorini, J. P., Fernandes, M. V., Decesaro, M. N., \& Marcon, S. S. (2008). A família no contexto hospitalar: apreendendo os anseios e expectativas relacionadas com doença crônica. Revista Rene, 3(2), 81-91.

Passos, S. S. S., Pereira, A., \& Nitschke, R. G. (2015). Cotidiano do familiar acompanhante durante a hospitalização de um membro da família. Acta Paulista de Enfermagem, 28(6), 539-545.

Perez, M. P., Silva, D. P. G., \& Couto, T. V. (2009). Percepções de um familiar de idoso hospitalizado na iminência de morte: um relato de caso. Arquivo Ciência Saúde, 16(1), 34-39.

Pena, S. B., \& Diogo, M. J. E. (2009). Expectativas da equipe de enfermagem e atividades realizadas por cuidadores de idosos hospitalizados. Revista da Escola de Enfermagem da USP, 43(2), 351-357.

Pinheiro, E. M., Silva, M. J. P., Angelo, M., \& Ribeiro, C. A. (2008). O significado da interação das profissionais de enfermagem com o recém/nascido família durante a hospitalização. Revista Latino Americana de Enfermagem, 16(6), 1012-1018.

Quintana, A. M., Wottrich, S. H., Camargo, V. P., Cherer, E. Q., \& Ries, P. K. (2011). Lutos e lutas: Reestruturações familiares diante do câncer em uma criança/adolescente, Psicologia Argumento, 29(65), 143-154.

Quirino, D. D., Collet, N., \& Neves, A. F. G. B. (2010). Hospitalização infantil: concepções da enfermagem acerca da mãe acompanhante. Revista Gaúcha de Enfermagem, 31(2), 300306.

Righetto-Dias, R., \& Nunes-Baptista, M. (2009). Medidas repetidas de suporte familiar e saúde mental em mães de crianças em UTI pediátrica. Universitas Psychology, 8(1), 125-136.

Roberts, M. C., \& Steele, R. G. (2009). Handbook of pediatric psychology (4th ed.). New York: Guilford Press.

Silva, S. G., Prochnow, A. G., Santos, J. L. G., Guerra, S. T., \& Barrios, S. G. (2009). The comunication between nursing team and the families of intensive care patients: qualitative study. Online Brazilian Journal of Nursing, 8(2), 33-45.

Silva, J. L., Santos, E. G. O., Rocha, C. C. T., Valença, C. N., \& Bay Júnior, O. G. (2015). Organização do trabalho de Enfermagem 
diante da inserção dos cuidados familiares com a criança hospitalizada. Revista RENE, 16(2), 226-232.

Sampaio, C. E. P., Silva, R. V., Comino, L. B. S., \& Romano, R. A. T. (2014). Nível de ansiedade dos acompanhantes de crianças em cirurgia ambulatorial: contribuições da consulta de enfermagem. Revista de Enfermagem UERJ, 22(2), 233-238.

Santos, A. M. R., Amorim, N. M. A., Braga, C. H., Lima, F. D. M., Macedo, E. M. A., \& Lima, C. F. (2011). Vivências de familiares de crianças internadas em um Serviço de Pronto-Socorro. Revista da Escola de Enfermagem da USP, 45(2), 473-479.

Santos, L. F., Oliveira, L. M. A. C., Barbosa, M. A., Siqueira, K. M., \& Peixoto, M. K. A. V. (2013). Reflexos da hospitalização da criança na vida do familiar acompanhante. Revista Brasileira de Enfermagem, 66(4), 473-478.

Schneider, D. G., Manschein, A. M. M., Ausen, M. A. B., \& Albuquerque, G. L. (2008). Acolhimento ao paciente e família na unidade coronariana. Texto Contexto Enfermagem, 17(1), 81-89.

Souza, E. (2011). A importância da família no tratamento do idoso hospitalizado. Revista da Sociedade de Cardiologia do Estado de São Paulo, 21 (Suplemento A), 29-34.

Souza, P., Scatolin, B. E., Ferreira, D. L. M., \& Croti, U. A. (2008). A relação da equipe de enfermagem com a criança e a família em pós-operatório imediato de cardiopatias congênitas. Arquivo Ciência Saúde, 15(4), 163-169.

Szareski, C., Beuter, M., \& Brondani, C. M. (2010). O familiar acompanhante no cuidado ao adulto hospitalizado na visão da equipe de enfermagem. Revista Gaúcha de Enfermagem, 31(4), 715-722.

Tyrrell, E. F., Levack, W. M., Ritchie, L. H., \& Keeling, S. M. (2012). Nursing contribution to the rehabilitation of older patients: patient and family perspectives. Journal of Advanced Nursing, 68(11), 2466-2476.

Urizzi, F., \& Corrêa, A. K. (2007). Vivências de familiares em terapia intensiva: o outro lado da internação. Revista latino-americana de enfermagem, 15(4), 598-604.

Vidal, V. L. L., Araujo, S. T. C., Perreault, M., \& Azevedo, A. L. (2013). O familiar acompanhante como estimulo comportamental de pacientes internados em terapia intensiva. Escola Anna Nery Revista de Enfermagem, 17(3), 409-415.

Verhaeghe, S. T. L., Van Zuuren, F.J.; Grypdonck, M. H., Duijnstee, M. S. H., \& Defloor, T. (2010). The focus of family members' functioning in the acute phase of traumatic coma. Journal of clinical nursing, 19(3-4), 574-582. 


\section{Endereço para correspondência}

Adriano Valério dos Santos Azevêdo

Universidade Federal de Santa Catarina

Laboratório de Psicologia da Saúde, Família e Comunidade (LABSFAC)

Centro de Filosofia e Ciências Humanas

Programa de Pós-Graduação em Psicologia - Campus Universitário Trindade

Caixa Postal 476, CEP 88040-900, Florianópolis - SC, Brasil

Endereço eletrônico: adrianoazevedopsi@yahoo.com

\section{Maria Aparecida Crepaldi}

Universidade Federal de Santa Catarina

Laboratório de Psicologia da Saúde, Família e Comunidade (LABSFAC)

Centro de Filosofia e Ciências Humanas

Programa de Pós-Graduação em Psicologia - Campus Universitário Trindade

Caixa Postal 476, CEP 88040-900, Florianópolis - SC, Brasil

Endereço eletrônico: maria.crepaldi@gmail.com

\section{Carmen Leontina Ojeda Ocampo More}

Universidade Federal de Santa Catarina

Laboratório de Psicologia da Saúde, Família e Comunidade (LABSFAC)

Centro de Filosofia e Ciências Humanas

Programa de Pós-Graduação em Psicologia - Campus Universitário Trindade

Caixa Postal 476, CEP 88040-900, Florianópolis - SC, Brasil

Endereço eletrônico: carmenloom@gmail.com

Recebido em: 05/02/2015

Reformulado em: 06/05/2016

Aceito para publicação em:26/05/2016

\section{Notas}

* Psicólogo, Mestre em Psicologia Social pela UFS/SE, Doutor em Psicologia pela UFSC/SC. Temas de pesquisa: Psicologia da Saúde, Psicologia Social Comunitária.

** Psicóloga, Mestre em Psicologia Clínica pela PUC/RJ, Doutora em Saúde Mental pela UNICAMP, pós-doutorado na Universidade do Québec em Montreal, Canadá e no HC/RP/USP. Temas de pesquisa: psicologia da saúde, psicologia da família.

*** Psicóloga, Mestrado e Doutorado em Psicologia Clínica pela PUC/SP. Áreas de pesquisa e interesse: família, violência familiar, redes sociais, psicologia da saúde, comunidade. 